\title{
Erratum to: COXIBRAIN: results of the prospective, randomised, phase II/III study for the selective COX-2 inhibition in chronic subdural haematoma patients
}

\author{
A. Schaumann ${ }^{1} \cdot$ W. Klene ${ }^{1} \cdot$ Chr. Rosenstengel ${ }^{2}$. \\ F. Ringel ${ }^{3} \cdot$ J. Tüttenberg ${ }^{4} \cdot$ P. Vajkoczy ${ }^{1}$
}

Published online: 9 November 2016

(C) Springer-Verlag Wien 2016

Erratum to: Acta Neurochir (2016) 158:2039-2044

DOI 10.1007/s00701-016-2949-3

The original version unfortunately contains incorrect affiliations for F. Ringel and J. Tüttenberg and is now corrected in this article.

The online version of the original article can be found at http://dx.doi. org/10.1007/s00701-016-2949-3.

A. Schaumann

Andreas.Schaumann@charite.de

1 Klinik für Neurochirurgie, Pädiatrische Neurochirurgie, Charité Campus Virchow Klinikum, Charité, Universitätsmedizin Berlin, Augustenburger Platz 1, 13353 Berlin, Germany

2 Körperschaft des öffentlichen Rechts - Klinik und Poliklinik für Neurochirurgie, Universitätsmedizin Greifswald, Sauerbruchstraße, 17475 Greifswald, Germany

3 Neurochirurgische Klinik und Poliklinik, Klinikum rechts der Isar der Technischen Universität München, Ismaninger Str. 22, 81675 München, Germany

4 Neurochirurgische Klinik, Universitätsmedizin Mannheim, Theodor-Kutzer-Ufer 1-3, 68167 Mannheim, Germany 